\title{
New constraints on the millimetre emission of six debris discs
}

\author{
Jonathan P. Marshall, ${ }^{1,2,3 \star}$ S. T. Maddison, ${ }^{4}$ E. Thilliez, ${ }^{4}$ B. C. Matthews,${ }^{5,6}$ \\ D. J. Wilner, ${ }^{7}$ J. S. Greaves ${ }^{8}$ and W. S. Holland ${ }^{9,10}$ \\ ${ }^{1}$ School of Physics, UNSW Australia, High Street, Kensington, Sydney, NSW 2052, Australia \\ ${ }^{2}$ Australian Centre for Astrobiology, UNSW Australia, High Street, Kensington, Sydney, NSW 2052, Australia \\ ${ }^{3}$ Computational Engineering and Science Research Centre, University of Southern Queensland, Toowoomba, QLD 4350, Australia \\ ${ }^{4}$ Centre for Astrophysics and Supercomputing, Swinburne University of Technology, Hawthorn, VIC 3122, Australia \\ ${ }^{5}$ National Research Council of Canada, 5071 West Saanich Rd, Victoria, BC V9E 2E7, Canada \\ ${ }^{6}$ Department of Physics and Astronomy, University of Victoria, Victoria, BC V8W 2Y2, Canada \\ ${ }^{7}$ Harvard-Smithsonian Center for Astrophysics, 60 Garden Street, Cambridge, MA 02138, USA \\ ${ }^{8}$ School of Physics and Astronomy, Cardiff University, Queen's Buildings, The Parade, Cardiff CF24 3AA, UK \\ ${ }^{9}$ UK Astronomy Technology Centre, Royal Observatory, Blackford Hill, Edinburgh EH9 3HJ, UK \\ ${ }^{10}$ Institute for Astronomy, University of Edinburgh, Royal Observatory, Blackford Hill, Edinburgh EH9 3 HJ, UK
}

Accepted 2017 March 13. Received 2017 March 13; in original form 2017 February 12

\begin{abstract}
The presence of dusty debris around main-sequence stars denotes the existence of planetary systems. Such debris discs are often identified by the presence of excess continuum emission at infrared and (sub-)millimetre wavelengths, with measurements at longer wavelengths tracing larger and cooler dust grains. The exponent of the slope of the disc emission at submillimetre wavelengths, ' $q$ ', defines the size distribution of dust grains in the disc. This size distribution is a function of the rigid strength of the dust producing parent planetesimals. As a part of the survey 'PLAnetesimals around TYpical Pre-main seqUence Stars', we observed six debris discs at $9 \mathrm{~mm}$ using the Australian Telescope Compact Array. We obtain marginal $(\sim 3 \sigma)$ detections of three targets: HD 105, HD 61005 and HD 131835. Upper limits for the three remaining discs, HD 20807, HD 109573 and HD 109085 provide further constraint of the (sub-)millimetre slope of their spectral energy distributions. The values of $q$ (or their limits) derived from our observations are all smaller than the oft-assumed steady-state collisional cascade model $(q=3.5)$, but lie well within the theoretically expected range for debris discs $q \sim 3-4$. The measured $q$ values for our targets are all $<3.3$, consistent with both collisional modelling results and theoretical predictions for parent planetesimal bodies being 'rubble piles' held together loosely by their self-gravity.
\end{abstract}

Key words: circumstellar matter-stars: individual: HD 105 -stars: individual: HD $20807-$ stars: individual: HD 61005 -stars: individual: HD 109085-stars: individual: HD $109573-$ stars: individual: HD 131835 - planetary systems.

\section{INTRODUCTION}

The debris discs observed around main-sequence stars are the remnants of primordial protoplanetary discs (Williams \& Cieza 2011; Wyatt et al. 2015), their constituent dust produced through the collisional attrition of asteroidal and cometary bodies or deposition by sublimation from comets (Wyatt 2008; Matthews et al. 2014). The size distribution of dust grains in a disc is observationally estimated by measuring the slope of the submillimetre emission from the disc (Beckwith et al. 1990; Gáspár et al. 2012). This slope is a function of the dust emissivity, stellar luminosity (Burns, Lamy \& Soter 1979) and the collisional state of the disc (Krivov 2010).
Collisional models of discs produce different predictions for the grain size distribution depending on the dynamical state or physical condition of the colliding planetesimals. In the steady-state scenario, a collisional cascade results from inelastic collisions and fragmentation that results in the dust grains following a power-law size distribution, that is, $\delta n \propto a^{-q} \delta a$, with a $q$ of 3.5 (Dohnanyi 1969). That standard model assumes a single constant tensile strength and velocity dispersion of the colliding bodies independent of their size. Such a scenario is unlikely to be true in nature.

In the Solar system, the size distribution of the largest bodies is a two-component power law with a break at a radius of $\sim 100 \mathrm{~km}$ (Bernstein et al. 2004, 2006; Fuentes \& Holman 2008; Fraser \& Kavelaars 2009; Fuentes, George \& Holman 2009). Above this radius the bodies have not been subjected to disruptive collisions and the size distribution exponent, $q=5$, is steeper than below it 
where $q=3$. For bodies below the break radius, the size distribution exponent is representative of collisional population whose binding energy is dominated by gravity (Pan \& Sari 2005). At smaller radii, around $0.1-1 \mathrm{~km}$, material strength dominates over gravitational binding energy and a different $q$ applies to the size distribution of those bodies (Benz \& Asphaug 1999). However, direct observation of Edgeworth-Kuiper belt bodies $<1 \mathrm{~km}$ in size is difficult due to the faintness of these bodies with typical albedos $\sim 6$ per cent. For the smallest grains, a size distribution exponent of $q=3$ is consistent with the non-detection of thermal emission from the disc (Vitense et al. 2012), although smaller $q$ values cannot be ruled out by the available data.

The assumed physical condition of dust parent bodies has a large impact on the derived size distribution of dust grains, varying from 3 to 4 for most models. As above, treating the dust-producing planetesimal bodies as 'rubble piles' held together by gravity with little internal strength produces $q$ values around 3.0-3.3 (Pan \& Sari 2005). The collisional modelling code 'Analysis of Collisional Evolution' (Krivov et al. 2013), with size-dependent grain strength, orientation and mutual gravity between colliding bodies, produces $q$ values 3.3-3.4 (Löhne et al. 2012; Schüppler et al. 2014, 2015). Including (one or more) refinements to the physics of the collisional modelling such as size-dependent tensile strength laws, mutual gravitation and size-dependent velocity distributions resulting from viscous stirring and collisional damping (Kenyon \& Bromley 2004; Pan \& Sari 2005; Löhne, Krivov \& Rodmann 2008; Gáspár et al. 2012; Pan \& Schlichting 2012) tends to produce steeper grain size distributions (higher $q$ ) of up to 3.8-4 when the tensile strength or velocity dispersion of the colliding bodies are steep functions of their size.

The theoretical expectation that $q$ will lie in the range 3-4 is borne out for the majority of discs. Observations at millimetre wavelengths using the Green Bank Telescope (Greaves et al. 2012), the Australian Telescope Compact Array (ATCA; Ricci et al. 2012, 2015) and the Jansky Very Large Array (MacGregor et al. 2016) demonstrated that most systems fell within the range of $3.0<q<4.0$. At far-infrared wavelengths, Pawellek et al. (2014) modelling of 34 resolved debris discs gave an error-weighted mean value of $q=$ $3.92 \pm 0.07$, whilst at millimetre wavelengths MacGregor et al. (2016) obtained an error-weighted value for $q=3.36 \pm 0.02$ from a sample of 15 discs. However, a number of pathological cases have been identified. For these discs, the values of $q$ determined from modelling of their spectral energy distributions (SEDs) lie well beyond the range of theoretically expected values. Such 'steep SED' discs identified at far-infrared and submillimetre wavelengths, e.g. Ertel et al. (2012), Montesinos et al. (2016) and Marshall et al. (2016), have $q$ values between 5 and 10, much higher than theoretical predictions.

The underlying nature of these discs with steep slopes is an open question. To better address this, determination of the slope values exhibited by discs across a broad range of spectral types and ages is required. Here, we report results from PLAnetesimals around TYpical and Pre-main seqUence Stars (PLATYPUS), an ongoing survey of debris discs at $9 \mathrm{~mm}$ with the ATCA. The targets in this work are six debris disc host stars spanning a broad range of stellar luminosities and ages.

In Section 2, we present the new observations made for the PLATYPUS survey, along with a summary of our data reduction and image analysis procedures. In Section 3, we present the results of our analysis, and combine these six targets with results from previously published phases of the PLATYPUS survey to examine the whole sample. In Section 4 , we discuss the survey results, both for individual targets and in aggregate. Finally, we present our conclusions and recommendations for future work in Section 5.

\section{OBSERVATIONS AND ANALYSIS}

\subsection{Observations}

A search for continuum emission at $9 \mathrm{~mm}$ from six debris discs was undertaken by ATCA using the Compact Array Broadband Backend digital filter bank (Wilson et al. 2011). These observations comprise the second part of the PLATYPUS survey (program ID C2694, P.I. Maddison), the previous results having been presented in Ricci et al. (2012, 2015). The targets selected here by and large are older and host fainter discs than those targets previously examined in comparable works (Ricci et al. 2015; MacGregor et al. 2016). A summary of relevant stellar parameters is given in Table 1. Positions are the stellar optical position in the International Coordinate Reference System at $J=2000$ epoch. Distances were taken from the re-reduction of the Hipparcos catalogue (van Leeuwen 2007). Ages were taken from the literature.

Predicted 9-mm flux densities for the targets ranged from 50 to $150 \mu \mathrm{Jy}$, and were based on modified blackbody models fitted to available far-infrared and submillimetre photometry. The integration times were between 290 and $360 \mathrm{~min}$, equivalent to an rms of $15 \mu \mathrm{Jy}$ beam $^{-1}$, chosen to ensure detection of the discs' emission based on extrapolations from available measurements. Apart from HD 61005, which has been observed at $9 \mathrm{~mm}$ (MacGregor et al. 2016), none of the targets have flux density measurements beyond $1.3 \mathrm{~mm}$. This is especially important for HD 105 and HD 20807, whose submillimetre SEDs are particularly poorly constrained. As an additional complication, HD 109085 hosts a large, extended disc and the total disc emission might therefore be resolved out with the array configuration used in these observations. The remaining discs were expected to be point-like at the resolution of our ATCA observations.

Observations were taken in 2-3-d spans covering 2015 April 4-6 (HD 109573, HD 109085 and HD 131835) and 2015 July 13-15 (HD 61005, HD 105 and HD 20807). All targets were observed using the compact $\mathrm{H} 214$ configuration, with baselines between 82 and $247 \mathrm{~m}$, giving a synthetic beam full width at half-maximum of $\sim 5 \operatorname{arcsec} \times 4 \operatorname{arcsec}$. This angular resolution is sufficient to resolve the nearest/largest discs in the sample, given sufficient signal to noise to interpret the emission. The ATCA comprises six 22-m antennas, one of which is located at a fixed position $6 \mathrm{~km}$ from the others. In our analysis, we have opted not to include data from the fixed antenna due to the increased phase noise on the much longer baselines. A summary of the observations is presented in Table 2.

Table 1. Stellar physical parameters.

\begin{tabular}{lcccccr}
\hline Target & $\begin{array}{c}\text { RA } \\
(\mathrm{h} \mathrm{m} \mathrm{s})\end{array}$ & $\begin{array}{c}\text { Dec. } \\
(\mathrm{d} \mathrm{m} \mathrm{s})\end{array}$ & $\begin{array}{c}d \\
(\mathrm{pc})\end{array}$ & $\begin{array}{c}\text { Sp. } \\
\text { Type }\end{array}$ & $\begin{array}{c}\text { Age } \\
(\mathrm{Myr})\end{array}$ & Ref. \\
\hline HD 105 & 000552.54 & -414511.0 & 39.4 & G0 V & 27 & 1,2 \\
HD 20807 & 031812.82 & -623022.9 & 12.0 & G0 V & 2000 & 3 \\
HD 61005 & 073547.46 & -321214.0 & 35.3 & G8 V & 90 & 4 \\
HD 109085 & 123204.22 & -161145.6 & 54.7 & F2 V & 1500 & 5 \\
HD 109573 & 123601.03 & -395210.3 & 72.8 & A0 V & 8 & 6 \\
HD 131835 & 145654.47 & -354143.7 & 122.7 & A2 V & 17 & 7 \\
\hline
\end{tabular}

Note. References: (1) Torres et al. (2000); (2) Zuckerman \& Webb (2000); (3) Eiroa et al. (2013); (4) Desidera et al. (2011); (5) Mallik, Parthasarathy \& Pati (2003); (6) Stauffer, Hartmann \& Barrado y Navascues (1995); (7) Moór et al. (2015). 
Table 2. Observation $\log$.

\begin{tabular}{llllll}
\hline $\begin{array}{l}\text { Date } \\
(2015)\end{array}$ & Target & Bandpass & $\begin{array}{l}\text { Calibrators } \\
\text { Flux }\end{array}$ & Phase & $\begin{array}{l}t_{\text {obs }} \\
(\mathrm{min})\end{array}$ \\
\hline Apr 4 & HD 109573 & $0537-441$ & $1934-638$ & $1144-379$ & 350 \\
Apr 5 & HD 109085 & $0537-441$ & $1934-638$ & $1213-172$ & 330 \\
Apr 6 & HD 131835 & $1424-418$ & $1934-638$ & $1451-375$ & 300 \\
Jul 13 & HD 61005 & $0537-441$ & $1934-638$ & $0745-330$ & 310 \\
Jul 14 & HD 105 & $1613-586$ & $1934-638$ & $2326-477$ & 290 \\
Jul 15 & HD 20807 & $1921-293$ & $1934-638$ & $0308-611$ & 390 \\
\hline
\end{tabular}

Table 3. ATCA photometric measurements.

\begin{tabular}{lccc}
\hline Target & $\begin{array}{c}\text { Beam size } \\
(\operatorname{arcsec})\end{array}$ & $\begin{array}{c}\text { Beam P.A. } \\
\left({ }^{\circ}\right)\end{array}$ & $\begin{array}{c}F_{9 \mathrm{~mm}^{a}} \\
\left(\mu \mathrm{Jy} \text { beam }^{-1}\right)\end{array}$ \\
\hline HD 105 & $5.0 \times 3.9$ & 79.4 & $42 \pm 14$ \\
HD 20807 & $5.2 \times 4.4$ & 76.5 & $<54$ \\
HD 61005 & $5.1 \times 3.9$ & 81.3 & $59 \pm 13$ \\
HD 109085 & $5.4 \times 4.0$ & 79.3 & $<36$ \\
HD 109573 & $5.1 \times 3.9$ & 79.2 & $<63$ \\
HD 131835 & $5.0 \times 3.8$ & 80.9 & $53 \pm 17$ \\
\hline
\end{tabular}

Note. ${ }^{a}$ Upper limits are $3 \sigma$.

\subsection{ATCA data reduction}

The ATCA observations were reduced in the MIRIAD software environment (version 1.5), following the recommended protocols for millimetre data as given in Chapter 4 of the ATCA Users Guide ${ }^{1}$. Flagging of radio frequency interference was undertaken using the package PGFLAG in the automated mode. The degree of radio frequency interference varied strongly with the baseline under consideration and the date of observation, but the fraction of discarded data was around 15-30 per cent. We used the standard packages INVERT, MFCLEAN and RESTOR to create images from each data set. Initially, the two sidebands taken of each target ( $2 \mathrm{GHz}$ bandwidth centred on 33 and $35 \mathrm{GHz}$ ) were reduced separately to examine each individually for reduction artefacts (e.g. from phase errors) or elevated noise. Finding none, we combined the two sidebands into a single map with a reference frequency of $34 \mathrm{GHz}(9 \mathrm{~mm})$ to reduce the overall noise of the final image. Source flux densities and uncertainties were measured using the package IMFIT, which fits a reconstructed point spread function to a given position in the image and derives the peak flux and residual noise.

\section{RESULTS}

We combined the newly acquired ATCA 9-mm photometry with observations from the literature spanning optical to millimetre wavelengths to produce SEDs for analysis. The optical data come from the Hipparcos catalogue (Perryman et al. 1997); near-infrared photometry are taken from the 2MASS (Skrutskie et al. 2006) and AllWISE (Wright et al. 2010) catalogues; mid- and far-infrared photometry were taken by Spitzer and Herschel (Donaldson et al. 2012; Eiroa et al. 2013; Duchêne et al. 2014; Moór et al. 2015; Morales et al. 2016); the (sub-)millimetre data were from Atacama Pathfinder Experiment (APEX), James Clerk Maxwell Telescope (JCMT), and Sub-Millimetre Array (SMA) (Nilsson et al. 2010; Ricarte et al. 2013; MacGregor et al. 2016). A summary of the ATCA photometry used in the disc modelling is presented in Table 3.

\footnotetext{
${ }^{1}$ https://www.narrabri.atnf.csiro.au/observing/users_guide/html/atug.html
}

The target spectra are presented in Fig. 1. ATCA images of the three detected disks (HD 105, HD 61005, and HD 131835) are presented in Fig. 2. The stellar component of the model was estimated by scaling a Castelli-Kurucz photospheric model (Castelli \& Kurucz 2004) of appropriate spectral type to optical and near-infrared photometry up to $10 \mu \mathrm{m}$, typically the $B V J H K_{s}$ and $W 3$ measurements. The disc contribution to the total flux was treated as a single temperature modified blackbody with a break wavelength $\lambda_{0}$ and exponent $\beta$ such that up to $\lambda_{0}$ the emission was blackbody-like, but beyond $\lambda_{0}$ the blackbody emission was multiplied by a factor of $\left(\lambda_{0} / \lambda\right)^{\beta}$. The disc temperature, $\lambda_{0}$, and $\beta$ were determined from a least-squares fit to the star-subtracted photometry beyond $50 \mu \mathrm{m}$ weighted by the uncertainties. For HD 109085 and HD 131835 , an additional warm blackbody component was used to fit the midinfrared emission prior to fitting the cold component, adopting the properties taken from Duchêne et al. (2014) and Moór et al. (2015), respectively.

Having fitted the target SEDs, we then proceeded to derive the exponent of the dust grain size distribution $q$ following MacGregor et al. (2016). To summarize briefly, we calculated the slope of a Planck function, $\alpha_{\mathrm{Pl}}$, from our observations at $9 \mathrm{~mm}$ and the next longest wavelength, typically $850 \mu \mathrm{m}$ or $1.3 \mathrm{~mm}$ :

$\alpha_{\mathrm{Pl}}=2+\frac{\log \left(\frac{2 k T_{\mathrm{d}}-h \nu_{1}}{2 k T_{\mathrm{d}}-h \nu_{2}}\right)}{\log \left(\nu_{1} / \nu_{2}\right)}$,

where $k$ is the Boltzmann constant, $h$ is the Planck constant, $T_{\mathrm{d}}$ is the disc temperature and $v_{1,2}$ are the frequencies of our two (sub-)millimetre observations. We also calculated the slope of the millimetre emission, $\alpha_{\mathrm{mm}}$ in a similar fashion:

$\alpha_{\mathrm{mm}}=\frac{\log \left(F_{v_{1}} / F_{v_{2}}\right)}{\log \left(v_{1} / v_{2}\right)}$,

where $F_{1,2}$ are the disc fluxes at the two frequencies of interest, $v_{1,2}$. Equation (2) assumes that both measurements used to derive the slope are in the Rayleigh-Jeans limit, which is valid for our debris discs. The value of $q$, the exponent of the grain size distribution, can be derived from these two quantities through the relation: $q=\left(\alpha_{\mathrm{mm}}-\alpha_{\mathrm{Pl}}\right) / \beta_{s}+3$. The relation holds for values of $q$ in the expected range of 3-4 that is typical for debris discs, and so should be valid here. In this relation, the $\beta_{s}$ parameter is taken from Draine (2006), who derived the relation between $\beta$ and $q$ for grains in protoplanetary discs. The value of $\beta_{s}$ is dependent on the assumed composition with a range of 1.3 , from material produced by the pyrolysis of cellulose at $800^{\circ} \mathrm{C}$ ('cel800'; Jaeger et al. 1994), to 2.0, derived for simple models of insulators and conductors (Draine 2004). Whilst values outside this range have been identified in specific cases (e.g. Bösch 1978; Agladze et al. 1996), their applicability to isolated small particles is not certain; small spheres are expected to have $\beta_{s} \geq 1.5$, but we here adopt a range $\beta_{s}$ of $1.3-2.0$ as a conservative approach (see Section 2 Draine 2006). For astronomical silicate, an oft-assumed debris dust material, the appropriate $\beta_{s}$ value is 1.8 and we use this value to calculate the $q$ values presented here. The range of $q$ for a disc resulting from selection of different $\beta_{s}$ values may be treated as a systematic uncertainty in the value of $q$, and are given separately in the table. The results of the disc fitting process and derived $q$ values are presented in Table 4.

Using this relation, we can also calculate the expected $q$ values for these discs based on the $\beta$ value from the modified blackbody fits. Rearranging the relation above we obtain $q=3+\left(\beta / \beta_{s}\right)$. For HD 105, the best-fitting value of $\beta=0.30 \pm 0.05$ is obtained. From this, we calculate that $q=3.17 \pm 0.03_{-0.04}^{+0.10}$, assuming a value of 

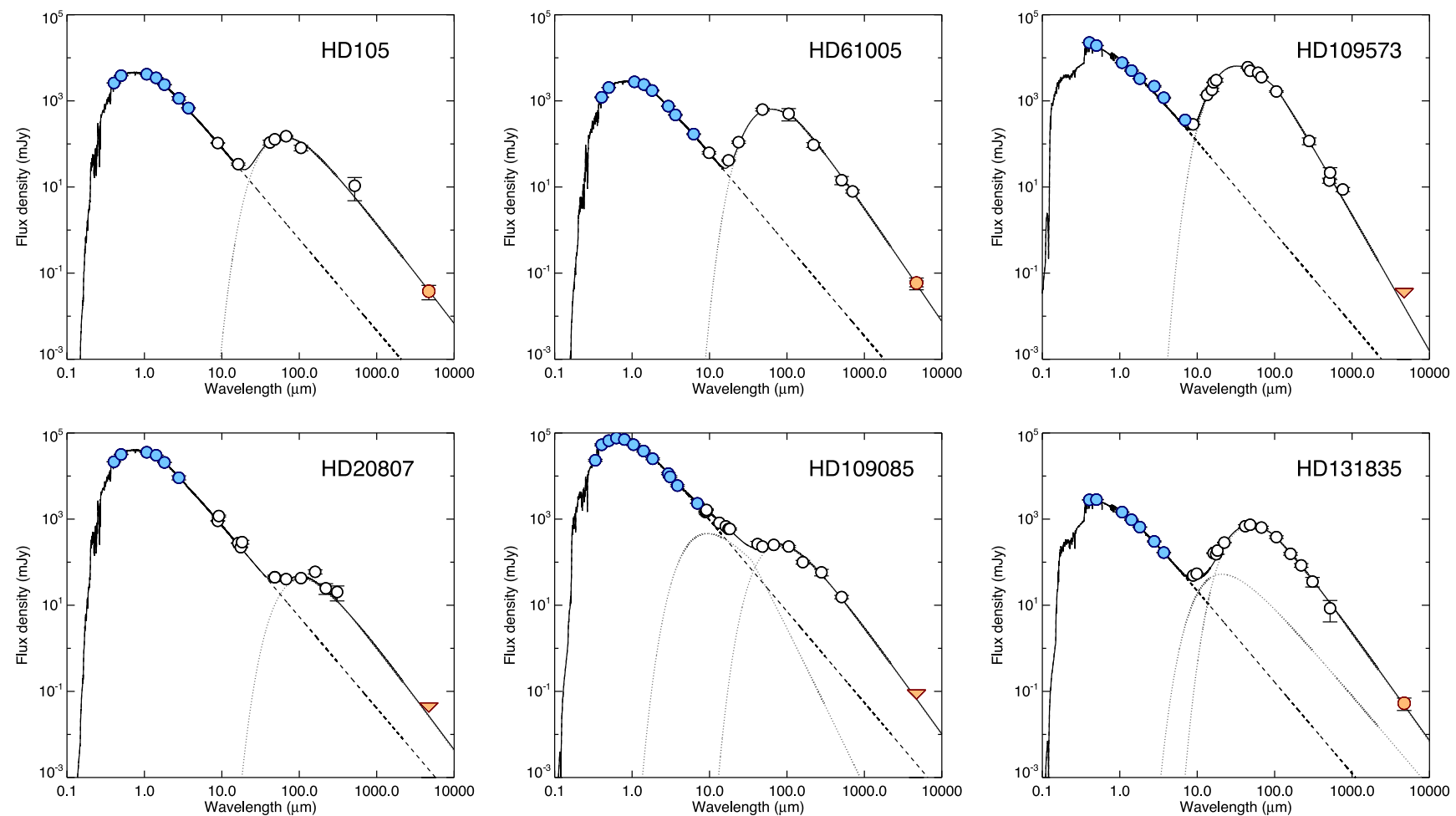

Figure 1. SEDs of the six debris discs examined in this work. White dots are ancillary optical and infrared photometry, as listed in Table 3 . The blue dots denote photometry used to scale the stellar photosphere for each system. The red dot/triangle denotes the ATCA 9-mm flux/3 $\sigma$ upper limit. The dashed grey line denotes the stellar photosphere, whilst the dotted grey line denotes the disc contribution(s). The black solid line is the total star+disc model.
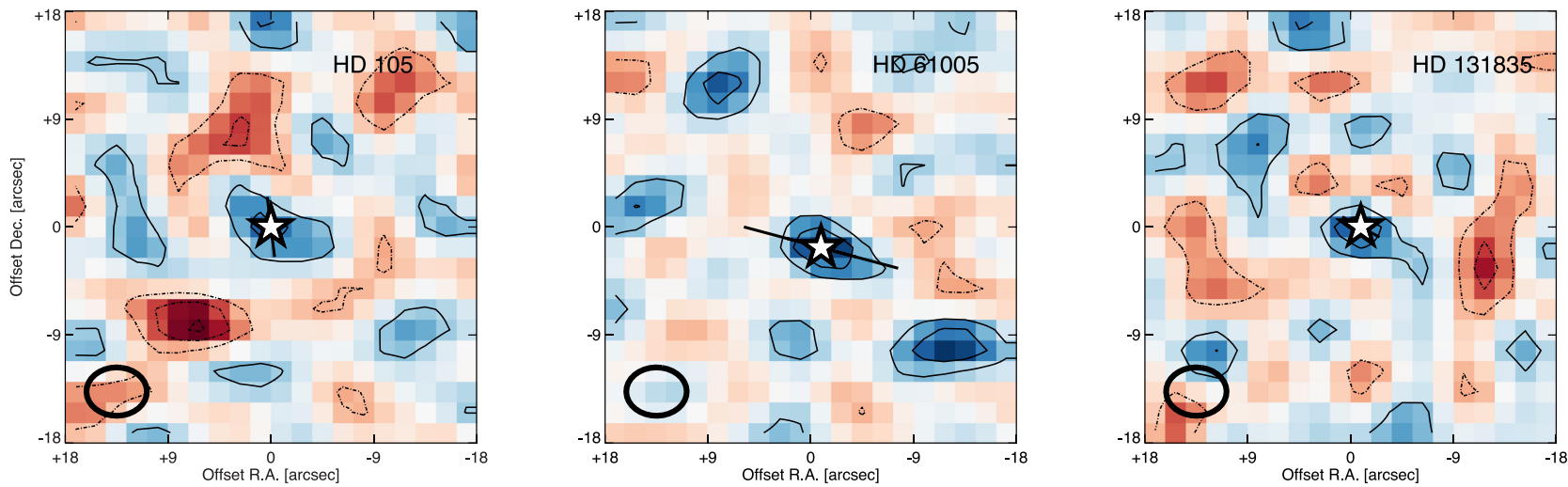

Figure 2. Images of the three debris discs detected in our 9-mm ATCA observations, left to right: HD 105, HD 61005 and HD 131835. The stellar position is denoted by the ' $\star$ ' symbol. The thick solid lines denote the disc extents and position angles, taken from Herschel (HD 105) or scattered light (HD 61005, HD 131835) imaging observations (Hines et al. 2007; Donaldson et al. 2012; Hung et al. 2015). The extension of HD 61005's disc along the expected position angle is not significant, given the low $\mathrm{s} / \mathrm{n}$ of the detection. Contours are in $1 \sigma$ increments from $\pm 2 \sigma$ and dotted lines denote negative flux. Colour scale is linear from $-3 \sigma$ to $+3 \sigma$. The ATCA beam ( $5 \operatorname{arcsec} \times 3 \operatorname{arcsec})$ is illustrated by the ellipse in the bottom-left corner. Orientation is north up, east left.

$\beta_{s}=1.8$ and a systematic component to the uncertainty coming from the range of possible values of $\beta_{s}$. Similarly, in the case of HD 131835, we find that $\beta=0.50 \pm 0.05$ and correspondingly $q=3.28 \pm 0.03_{-0.06}^{+0.14}$.

\section{DISCUSSION}

\subsection{Individual results}

\subsubsection{HD 105}

HD 105 is a young G0 V star at a distance of $40 \mathrm{pc}$ that hosts a moderately bright debris disc $\left(L_{\text {dust }} / L_{\star} \sim 2.6 \times 10^{-4}\right)$. As a member of the Tucana-Horologium association (Torres et al. 2000; Zuckerman \& Webb 2000), the system's age of $30 \mathrm{Myr}$ is reasonably well determined. Its debris disc was marginally resolved in farinfrared Herschel/PACS imaging observations and found to have a radius of $52 \mathrm{au}$ (Donaldson et al. 2012). HD 105 might therefore be considered an analogue of the young Sun. The solitary submillimetre photometric point at $870 \mu \mathrm{m}$ from APEX (Nilsson et al. 2010) only provides weak constraint on the disc submillimetre emission, and lies $1 \sigma$ above the best-fitting model obtained with our new 9mm flux measurement of $42 \pm 14 \mu \mathrm{Jy}$. A value of $q=3.26 \pm 0.17$ was derived for the grain size distribution exponent for this disc. 
Table 4. Grain size distribution slopes, $q$.

\begin{tabular}{lccccccr}
\hline Target & $\begin{array}{c}\lambda_{\text {(sub-)mm }} \\
(\mathrm{mm})\end{array}$ & Instrument & \multicolumn{1}{c}{$\begin{array}{c}\text { Flux } \\
(\mathrm{mJy})\end{array}$} & Reference & $\alpha_{\mathrm{mm}}$ & $\alpha_{\mathrm{Pl}}$ & $q$ \\
\hline HD 105 & 0.87 & APEX & $10.7 \pm 5.9$ & 1 & $2.41 \pm 0.16$ & $1.94 \pm 0.07$ & $3.26 \pm 0.17_{-0.03}^{+0.10}$ \\
HD 20807 & 0.50 & SPIRE & $20.3 \pm 7.7$ & 2 & $2.05 \pm 0.10$ & $1.79 \pm 0.28$ & $>3.15^{+0.09}$ \\
HD 61005 & 1.30 & SMA & $7.2 \pm 1.5$ & 3 & $2.48 \pm 0.08$ & $2.05 \pm 0.05$ & $3.24 \pm 0.09_{-0.02}^{+0.05}$ \\
HD 109085 & 0.85 & SCUBA2 & $14.3 \pm 1.1$ & 4 & $2.10 \pm 0.07$ & $1.90 \pm 0.12^{2}$ & $>3.11^{2}$ \\
HD 109573 & 0.85 & SCUBA2 & $14.4 \pm 1.9$ & 4 & $2.73 \pm 0.10$ & $1.98 \pm 0.02$ & $>3.42^{+0.05}$ \\
HD 131835 & 0.87 & APEX & $8.5 \pm 4.4$ & 5 & $2.17 \pm 0.13$ & $1.95 \pm 0.05$ & $3.12 \pm 0.14_{-0.01}^{+0.05}$ \\
\hline
\end{tabular}

Notes. Values of $\alpha_{\mathrm{Pl}}$ and $\alpha_{\mathrm{mm}}$ for targets with non-detections at $9 \mathrm{~mm}$ were calculated based on the $3 \sigma$ upper limit.

References: (1) Nilsson et al. (2010); (2) Eiroa et al. (2013); (3) Ricarte et al. (2013); (4) Holland et al. (in preparation); (5) Moór et al. (2015).

\subsubsection{HD 20807}

HD 20807 is another nearby Sun-like star (G1 V), lying at 12 pc. It was identified as a debris disc host star and thought to be resolved by Herschel/PACS observations (Eiroa et al. 2013). The putative disc is thought to be non-axisymmetric, potentially due to the presence of a substellar companion on a wide orbit (Faramaz et al. 2014). Herschel/SPIRE measurements in the submillimetre reveal a flat spectrum out to $\sim 500 \mu \mathrm{m}$, offering no clues as to the point of turnover or steepness of the decline in disc emission. Subsequent analysis of the same Herschel data has cast doubt upon the interpretation of HD 20807 as a debris disc host star, with Moro-Martín et al. (2015) finding no evidence for a far-infrared excess. We obtained a $3 \sigma$ upper limit of $54 \mu \mathrm{Jy}_{\text {beam }^{-1}}$ from the ATCA measurements, and a corresponding lower limit of $q>3.15$. The beam size of ATCA in a compact mode and Herschel/PACS are comparable, so the non-detection of this target may be due to dilution of the total disc flux over multiple beams or, potentially, due to the absence of any disc around the star in the first place.

\subsubsection{HD 61005}

HD 61005 (the moth) is a very well-studied young debris disc system around a late G-type star at a distance of $35 \mathrm{pc}$. Its disc is bright, having been imaged in scattered light (Hines et al. 2007), and resolved at millimetre wavelengths (Ricarte et al. 2013; MacGregor et al. 2016). The disc exhibits a pronounced asymmetry that has been attributed to either interaction with the interstellar medium (Maness et al. 2009), or the presence of planetary companion perturbing the disc (Buenzli et al. 2010; Esposito et al. 2016). We obtained a $9-\mathrm{mm}$ flux density of $59 \pm 14 \mu \mathrm{Jy}$ for this system. This is completely consistent with the 9-mm flux obtained by MacGregor et al. (2016). Using our 9-mm measurement in combination with the SCUBA-2 flux density at $850 \mu \mathrm{m}$, we obtained $q=3.08 \pm 0.17$ for the disc. This is marginally steeper than the earlier derivation of (MacGregor et al. 2016, $q=3.32 \pm 0.06$ ) using the 1.3-mm SMA flux density (7.2 $\pm 1.5 \mathrm{mJy})$ to calculate $\alpha_{\mathrm{mm}}$, for which we obtain $q=3.24 \pm 0.09$ (as presented in Table 4$)$.

\subsubsection{HD 109085}

HD 109085 ( $~ \eta$ Crv), a 1.5-Gyr-old F2 V star at a distance of 55 pc, hosts a disc with an inner warm component that is anomalously bright for its age. The disc was resolved into two distinct components by Herschel/PACS (Duchêne et al. 2014). The outer belt was recently resolved at millimetre wavelengths by Atacama Large Millimetre/sub-millimetre Array observations that also identified $\mathrm{CO}$ emission from the inner regions of the disc (Marino et al. 2017). This places it (along with HD 131835) amongst the small number of debris discs that also have detectable gas (Greaves et al. 2016). Interpretation of its mid-infrared spectrum reveals evidence of primordial material, believed to originate from icy planetesimals scattered from the outer belt to the inner regions of the system (Lisse et al. 2012). We obtained an upper limit of $36 \mu \mathrm{Jy}^{\text {beam }^{-1}}$ with ATCA. Due to the extended nature of the source, covering an area of $\sim 3$ beams, the outer belt's surface brightness is too low to be detectable with the sensitivity of ATCA. However, our measurement was a meaningful constraint to the outer disc as resolved in Marino et al. (2017). We thus obtained a lower limit of $q>3.11$; this value was calculated taking into account the area of the disc.

\subsubsection{HD 109573}

HD 109573 (HR 4796 A), an 8-Myr-old A-type star at 73 pc, is the most distant of our targets. It was the second disc to be spatially resolved (after $\beta$ Pictoris) being imaged at mid-infrared wavelengths (Jayawardhana et al. 1998; Koerner et al. 1998). The disc has also been imaged successfully in scattered light, being dubbed the 'eye of Sauron' (Schneider et al. 2009). The disc has a narrow radial width $(\Delta R / R \sim 14$ per cent; Rodigas et al. 2015) and its structure has been interpreted as indicating the presence of a shepherding planetary companion (Thalmann et al. 2011). Given that the disc's emission is well sampled, and its small angular extent, we expected that this system would yield a clear detection in our 9-mm observations. However, we only obtained a $3 \sigma$ upper limit of $63 \mu \mathrm{Jy}_{\text {beam }}{ }^{-1}$ equating to a limit on $q>3.42$. This is well within the expected range of 3-4 for debris discs. We attribute the non-detection of this system to the elevated millimetre photometry from APEX $(21.5 \pm 6.6$ mJy; Nilsson et al. 2010) and JCMT/SCUBA (19.1 $\pm 3.4 \mathrm{mJy}$; Greaves, Mannings \& Holland 2000) compared to the more recent JCMT/SCUBA2 measurement (14.4 $\pm 1.9 \mathrm{mJy}$; Holland, in preparation) that biased the modified blackbody fit.

\subsubsection{HD 131835}

HD 131835 is a young (17 Myr old), massive analogue to the $\beta$ Pictoris system. Submillimetre observations of the disc detected the presence of CO gas in the disc (Moór et al. 2015), making it only one of a handful of such systems (see e.g. Greaves et al. 2016). It has recently been imaged in scattered light by Gemini Planet Imager, revealing a broad (75-210 au), inclined (75 ) disc around the host star (Hung et al. 2015). The disc was also marginally resolved by Herschel/PACS far-infrared observations, suggesting an extent of 170 au (Moór et al. 2015). Here, we obtained the first measurement of the disc continuum emission $9 \mathrm{~mm}$, with a flux of $53 \pm 17 \mu \mathrm{Jy}$. The corresponding $q=3.12 \pm 0.14$. This disc has the shallowest submillimetre slope amongst the discs measured here; this could be 
Table 5. Comparison of $q$ values derived by the method of Gáspár et al. (2012) and in this work.

\begin{tabular}{lcc}
\hline Target & $q_{\text {Gaspar }}$ & $q_{\text {Thiswork }}$ \\
\hline$\beta$ Pic & $3.63_{-0.26}^{+0.24}$ & $3.53 \pm 0.10$ \\
$\epsilon$ Eri & $3.14_{-0.14}^{+0.49}$ & $2.53 \pm 0.18$ \\
$\alpha$ PsA & $3.62_{-0.20}^{+0.26}$ & $3.21 \pm 0.07$ \\
HD 8907 & $3.00_{-0.00}^{+0.56}$ & $2.64 \pm 0.20$ \\
HD 104860 & $3.07_{-0.07}^{+0.57}$ & $2.70 \pm 0.22$ \\
HD 107146 & $3.34_{-0.34}^{+0.42}$ & $3.11 \pm 0.12$ \\
HR 8799 & $3.21_{-0.31}^{+0.33}$ & $4.11 \pm 0.33$ \\
$\alpha$ Lyr & $4.01_{-0.28}^{+0.30}$ & $3.61 \pm 0.17$ \\
HD 207129 & $3.42_{-0.42}^{+0.65}$ & $3.74 \pm 0.38$ \\
\hline
\end{tabular}

attributed to the influence of gas in the disc moderating the collisions between dust grains.

\subsection{Comparison with previous work}

The $q$ values obtained for the newly detected discs were smaller than the average value for mm-detected discs calculated in MacGregor et al. (2016) (3.36 \pm 0.02$)$, and that of the commonly adopted steady-state collisional cascade of Dohnanyi (1969). Combining values for the three detected discs in the sample, we found an errorweighted $q$ of $3.15 \pm 0.09$. If we add the three non-detections (treating the $3 \sigma$ upper limit at $9 \mathrm{~mm}$ as a detection), the mean $q$ increases to $3.26 \pm 0.05$, and is still smaller than the value of MacGregor et al. (2016). The lower sensitivity of ATCA compared to Jansky Very Large Array (VLA) at the same wavelength would account for our detection of discs with smaller $q$ values, as these discs have a greater contribution from larger grains to their total emission and would therefore be relatively brighter.

At first glance, the values are also discrepant with those obtained by Gáspár et al. (2012). An assumption of that work was that millimetre emission from the disc is dominated by grains with $Q_{\mathrm{abs}}=1$, such that the relationship between $q$ and $\alpha_{\mathrm{mm}}$ is $q=\alpha_{\mathrm{mm}}+1$. This is contrary to the results of Draine (2006). We have recalculated the values of $q$ for the nine discs examined in Gáspár et al. (2012) using the same method employed here and in MacGregor et al. (2016). A side-by-side comparison of the derived values for $q$ using the same photometry is given in Table 5 .

In all cases except two, the values of $q$ are found to be smaller using the method applied here and in MacGregor et al. (2016) than by that of Gáspár et al. (2012). In combination, the revised disc measurements have a mean $q=3.23 \pm 0.04$. The revised values we obtain are more in line with the findings of MacGregor et al. (2016) and numerical results (e.g. Schüppler et al. 2014, 2015) than with the modelling results of far-infrared excesses (Pawellek et al. 2014). In the two instances where our method yields a steeper $q$ than Gaspar et al., that is, HR 8799 and HD 207129, we note that the longer wavelength of the submillimetre SED used to calculate $\alpha_{\mathrm{mm}}$ is poorly constrained, being represented by sub- $2 \sigma$ detections in each case; better quality submillimetre photometry would likely yield a flatter size distribution (smaller $q$ ).

Three of the discs, $\epsilon$ Eri, HD 8907 and HD 104860, have $q$ values $<3$. These are outside the generally expected range of $3-4$ for debris discs, and beyond the regime where the relations derived in Draine (2006) were found to hold. In MacGregor et al. (2016) they identified one system, HD 141569, with similar properties $(q=2.84 \pm 0.05)$. They attributed its small size distribution ex- ponent to the fact that it was a young, gas-rich protoplanetary disc system unlike the debris discs that made up the majority of their sample. Here, such a line of reasoning does not hold as all three are well-known debris disc systems.

We therefore investigated the impact of more recent, higher quality photometry than that presented in Gáspár et al. (2012). Using recent millimetre photometry from the SMA (Steele et al. 2016) and LMT (Chavez-Dagostino et al. 2016), we recalculated the values of these three discs and found them consistent with the remainder of the sample, with $q=3.21 \pm 0.31$ ( $\epsilon$ Eri), $3.69 \pm 0.23$ (HD 8907) and $3.26 \pm 0.59$ (HD 104860). With the revised measurements, the mean $q$ value of Gaspar et al.'s sample changes to $3.35 \pm 0.05$, completely consistent with MacGregor et al. (2016).

\section{CONCLUSIONS}

We set out to measure the continuum emission from six debris discs at millimetre wavelengths using the ATCA in order to constrain the slope of their submillimetre emission and thereby better determine the planetesimal properties in those systems. Our observations have demonstrated the capability of ATCA to detect continuum emission from relatively faint debris discs $\left(L_{\text {dust }} / L_{\star}<10^{-3}\right)$ that are most representative of the bulk of known, cool circumstellar discs.

We detected three of the six discs at $\mathrm{s} / \mathrm{n}>3$ in our observations. Two of the targets, HD 105 and HD 131835, were detected for the first time at millimetre wavelengths. HD 61005 had been previously observed at $9 \mathrm{~mm}$ with a comparable flux density to the measurement presented here. Upper limits for the remaining three targets (HD 20807, HD 109085 and HD 109573) placed strong constraints on the slope of the discs' submillimetre emission.

We found that the exponents of the size distribution, $q$, for the three detected discs were smaller than both the steady-state collisional cascade model of Dohnanyi (1969), and also smaller than the mean $q$ value obtained for the sample of 15 debris discs examined in MacGregor et al. (2016). For the detected discs, the $q$ values are most consistent with theoretical models wherein the planetesimals are treated as 'rubble piles' without any rigid strength (Pan \& Sari 2005), and for the most part consistent with collisional modelling results of Löhne et al. (2012) and Schüppler et al. (2014, 2015). The lower limits on the $q$ values of HD 20807 and HD 109085 are both high enough that we cannot rule out consistency with MacGregor et al. (2016), whilst the upper limit on HD 109573's $q$ value is low enough such that the disc grain size distribution must be steeper than is typical for millimetre-detected discs. Given the brightness of HD 109573's disc at submillimetre wavelengths and its relatively compact nature, further study at comparable wavelengths and higher sensitivity (e.g. 9-mm observations on the Jansky VLA) should be expected to yield a detection of the disc.

\section{ACKNOWLEDGEMENTS}

We would like to thank the referee for his/her careful and considerate comments that improved the manuscript. JPM would like to thank Paul Jones for helpful discussions on the ATCA data reduction process. JPM is supported by a UNSW Vice-Chancellor's Postdoctoral Research Fellowship. This research has made use of the SIMBAD data base, operated at CDS, Strasbourg, France. This research has made use of NASA's Astrophysics Data System. The Australia Telescope Compact Array is part of the Australia Telescope National Facility, which is funded by the Australian Government for operation as a National Facility managed by CSIRO.

Facilities: ATCA 


\section{REFERENCES}

Agladze N. I., Sievers A. J., Jones S. A., Burlitch J. M., Beckwith S. V. W., 1996, ApJ, 462, 1026

Beckwith S. V. W., Sargent A. I., Chini R. S., Guesten R., 1990, AJ, 99, 924 Benz W., Asphaug E., 1999, Icarus, 142, 5

Bernstein G. M., Trilling D. E., Allen R. L., Brown M. E., Holman M., Malhotra R., 2004, AJ, 128, 1364

Bernstein G. M., Trilling D. E., Allen R. L., Brown M. E., Holman M., Malhotra R., 2006, AJ, 131, 2364

Bösch M. A., 1978, Phys. Rev. Lett., 40, 879

Buenzli E. et al., 2010, A\&A, 524, L1

Burns J. A., Lamy P. L., Soter S., 1979, Icarus, 40, 1

Castelli F., Kurucz R. L., 2004, preprint (arXiv:e-prints)

Chavez-Dagostino M. et al., 2016, MNRAS, 462, 2285

Desidera S. et al., 2011, A\&A, 529, A54

Dohnanyi J. S., 1969, J. Geophys. Res., 74, 2531

Donaldson J. K. et al., 2012, ApJ, 753, 147

Draine B. T., 2004, Astrophysics of Dust in Cold Clouds. Springer-Verlag, Berlin, p. 213

Draine B. T., 2006, ApJ, 636, 1114

Duchêne G. et al., 2014, ApJ, 784, 148

Eiroa C. et al., 2013, A\&A, 555, A11

Ertel S. et al., 2012, A\&A, 541, A148

Esposito T. M. et al., 2016, AJ, 152, 85

Faramaz V. et al., 2014, A\&A, 563, A72

Fraser W. C., Kavelaars J. J., 2009, AJ, 137, 72

Fuentes C. I., Holman M. J., 2008, AJ, 136, 83

Fuentes C. I., George M. R., Holman M. J., 2009, ApJ, 696, 91

Gáspár A., Psaltis D., Rieke G. H., Özel F., 2012, ApJ, 754, 74

Greaves J. S., Mannings V., Holland W. S., 2000, Icarus, 143, 155

Greaves J. S., Hales A. S., Mason B. S., Matthews B. C., 2012, MNRAS, 423, L70

Greaves J. S. et al., 2016, MNRAS, 461, 3910

Hines D. C. et al., 2007, ApJ, 671, L165

Hung L.-W. et al., 2015, ApJ, 815, L14

Jaeger C., Mutschke H., Begemann B., Dorschner J., Henning T., 1994, A\&A, 292, 641

Jayawardhana R., Fisher S., Hartmann L., Telesco C., Piña R., Fazio G., 1998, ApJ, 503, L79

Kenyon S. J., Bromley B. C., 2004, AJ, 127, 513

Koerner D. W., Ressler M. E., Werner M. W., Backman D. E., 1998, ApJ, 503, L83

Krivov A. V., 2010, Res. Astron. Astrophys., 10, 383

Krivov A. V. et al., 2013, ApJ, 772, 32

Lisse C. M. et al., 2012, ApJ, 747, 93

Löhne T., Krivov A. V., Rodmann J., 2008, ApJ, 673, 1123

Löhne T. et al., 2012, A\&A, 537, A110

MacGregor M. A. et al., 2016, ApJ, 823, 79
Mallik S. V., Parthasarathy M., Pati A. K., 2003, A\&A, 409, 251

Maness H. L. et al., 2009, ApJ, 707, 1098

Marino S. et al., 2017, MNRAS, 465, 2595

Marshall J. P., Booth M., Holland W., Matthews B. C., Greaves J. S., Zuckerman B., 2016, MNRAS, 459, 2893

Matthews B. C., Krivov A. V., Wyatt M. C., Bryden G., Eiroa C., 2014, Protostars and Planets VI. Univ. Arizona Press, Tucson, AZ, p. 521

Montesinos B. et al., 2016, A\&A, 593, A51

Moór A. et al., 2015, ApJ, 814, 42

Morales F. Y., Bryden G., Werner M. W., Stapelfeldt K. R., 2016, ApJ, 831, 97

Moro-Martín A. et al., 2015, ApJ, 801, 143

Nilsson R. et al., 2010, A\&A, 518, A40

Pan M., Sari R., 2005, Icarus, 173, 342

Pan M., Schlichting H. E., 2012, ApJ, 747, 113

Pawellek N., Krivov A. V., Marshall J. P., Montesinos B., Ábrahám P., Moór A., Bryden G., Eiroa C., 2014, ApJ, 792, 65

Perryman M. A. C. et al., 1997, A\&A, 323, L49

Ricarte A., Moldvai N., Hughes A. M., Duchêne G., Williams J. P., Andrews S. M., Wilner D. J., 2013, ApJ, 774, 80

Ricci L., Testi L., Maddison S. T., Wilner D. J., 2012, A\&A, 539, L6

Ricci L., Maddison S. T., Wilner D., MacGregor M. A., Ubach C., Carpenter J. M., Testi L., 2015, ApJ, 813, 138

Rodigas T. J. et al., 2015, ApJ, 798, 96

Schneider G., Weinberger A. J., Becklin E. E., Debes J. H., Smith B. A., 2009, AJ, 137, 53

Schüppler C., Löhne T., Krivov A. V., Ertel S., Marshall J. P., Eiroa C., 2014, A\&A, 567, A127

Schüppler C. et al., 2015, A\&A, 581, A97

Skrutskie M. F. et al., 2006, AJ, 131, 1163

Stauffer J. R., Hartmann L. W., Barrado y., Navascues D., 1995, ApJ, 454, 910

Steele A., Hughes A. M., Carpenter J., Ricarte A., Andrews S. M., Wilner D. J., Chiang E., 2016, ApJ, 816, 27

Thalmann C. et al., 2011, ApJ, 743, L6

Torres C. A. O., da Silva L., Quast G. R., de la Reza R., Jilinski E., 2000, AJ, 120, 1410

van Leeuwen F., 2007, A\&A, 474, 653

Vitense C., Krivov A. V., Kobayashi H., Löhne T., 2012, A\&A, 540, A30

Williams J. P., Cieza L. A., 2011, ARA\&A, 49, 67

Wilson W. E. et al., 2011, MNRAS, 416, 832

Wright E. L. et al., 2010, AJ, 140, 1868

Wyatt M. C., 2008, ARA\&A, 46, 339

Wyatt M. C., Panić O., Kennedy G. M., Matrà L., 2015, Ap\&SS, 357, 103

Zuckerman B., Webb R. A., 2000, ApJ, 535, 959

This paper has been typeset from a $\mathrm{T}_{\mathrm{E}} \mathrm{X} / \mathrm{LAT} \mathrm{E}$ f file prepared by the author. 